\title{
The Role of Healthcare Personnel in Delivering Healthcare Interventions for High Blood Pressure: A Desk Review of Available Evidence
}

\author{
Subhana Akber Khan ${ }^{1 *}$, Saadullah Afridi ${ }^{2}$, Ejaz Ahmad Khan ${ }^{1}$, Zeeshan Kibria ${ }^{2}$, Siham \\ Sikander ${ }^{1}$, Syed Fawad Mashadii ${ }^{1}$ and Assad Hafeez ${ }^{1}$ \\ ${ }^{1}$ PhD Fellow, Health Services Academy, Pakistan \\ ${ }^{2}$ Dean \& Director, Sarhad Institute of Health Sciences, Sarhad University, Pakistan
}

*Corresponding author: Subhana Akber Khan, Health Services Academy, Islamabad, Pakistan.

To Cite This Article: Subhana Akber K, Saadullah A, Ejaz Ahmad K, Zeeshan K, Siham S, et al., The Role of Healthcare Personnel in Delivering Healthcare Interventions for High Blood Pressure: A Desk Review of Available Evidence. Am J Biomed Sci \& Res. 2021 - 12(1). AJBSR. MS.ID.001706. DOI: 10.34297/AJBSR.2021.12.001706.

Received: 眥 February 11, 2021; Published: 笽 February 16, 2021

\begin{abstract}
Objective: The objective of this study is to determine various healthcare personnel in delivering interventions as recommended by Disease Control Priorities (DCP3) strategies to manage and control high blood pressure among hypertensive patients.

Methods: The guidance for conducting this desk review was adapted from a toolkit published by WHO and UNHCR (2012) and Save the Children. Secondary analysis was conducted of academically available and existing literature regarding hypertension management and control from 2015 to 2019 using PubMed and Google Scholar. Qualitative analysis of 526 articles was performed as per eligibility criteria and content of the desk review was prepared in accordance with the study objective by synthesizing available evidence.
\end{abstract}

Results: Available evidence for blood pressure reduction interventions delivered by physicians, pharmacists, nurses and other health professionals were included. Five key components of DCP3 strategies were focused which comprised of interventions mainly for sodium intake $(n=140)$, smoking cessation $(n=160)$, physical activity, obesity and BMI $(n=226)$. The findings elucidate that interventions for sodium intake by physicians are mostly studied worldwide for lowering blood pressure followed by smoking cessation and physical activity for hypertension. Less available data exists for nurses; however, pharmacists and CHWs are being mainly included for delivering supervised interventions and in task shifting roles.

Conclusion: Control of blood pressure has been studied extensively within service delivery domain of DCP3 interventions. Although, most of the interventions have been physician led but for hypertension management, population-level interventions are needed for prevention of non-communicable diseases. Rural population must also be focused in implementing BP reduction strategies that are locally adapted. Whereas considering understaffed health system in LMICs, other healthcare professionals can be trained and supervised to implement awareness and behavioral interventions in communities.

Keywords: High blood pressure; Intervention; Disease Control Priorities; Healthcare

\section{Introduction}

Hypertension is worldwide major cause of morbidity and mortality due to cardiovascular diseases. Globally, hypertension is responsible for 300 million years of life lost (YLL) and cause to contribute more than 35 million years for disability adjusted life years (DALYs) [1]. High blood pressure causing hypertension can be prevented and is treatable, but it is accounted for major loss to life, and costly health outcomes such as stroke, renal diseases and other complications [2]. According to United Nations (UN) one of the key targets is to reduce premature deaths by $25 \%$ from non-communicable diseases by 2025 . To meet UN target by 2025 , rigorous researches are needed to address the gaps related to potential risk factors owing to pre-mature mortality (which is the probability of dying between ages of 30-70 years). These risk factors largely are obesity, use of tobacco, diabetes mellitus 
and plasma glucose levels. In high-income countries, reduction in the body mass index (BMI) has been primarily implicated in reducing systolic blood pressure (SBP). In South Asia, the projected premature deaths from cardiovascular diseases because of the presence of related risk factors are expected to rise by $56 \%$ due to growth and aging of population. However, evidence suggests that strategies addressing multiple risk factors are found to be effective concerning all regions of the world [3]. No significant change has been observed in high-income countries for cardiovascular diseases (CVDs) but a $66 \%$ increase in deaths has been witnessed in low-and middle-income (LMICs) due to cardiovascular diseases as compared to high-income countries [4]. DCP3 suggests multilevel strategies for hypertension control but the pharmacologic recommendations to avoid adverse effects on the body weight are not specified in hypertension guidelines [5]. Prevention of hypertension leads to reduced onset of cardiovascular events as evidence suggests that pretreatment and assessment of CVD risk of patients suffering from high blood pressure across the spectrum of blood pressure shows beneficial results [6].

Generally, reduction in blood pressure among masses can be achieved when multiple sectors will engage in accordance with country-specific priorities to tackle CVD related morbidity and mortality. Collaboration between public-private partnerships as well as population-based strategies that are cost-effective are needed to be implemented to yield achievable success [3]. In addition, government-led strategies and policy if combined with collaboration with industrial sector are most cost-effective. Such integrated and health system-based initiatives for NCDs if introduced to raise awareness and educate individuals and patients regarding hypertension can help to save limited healthcare spending particularly in the context of LMICs [7]. The study aimed to identify interventions used for blood pressure reduction among hypertensive patients and to ascertain the gaps in practices, key challenges and opportunities available to refine development of DCP3 based intervention for hypertension management and control. Rationale for conducting a desk review on DCP3 based interventions including pharmacological and non-pharmacological strategies for hypertension management and control among hypertensive patients is to: i) identify available evidence on hypertension related interventions and strategies to reduce blood pressure, ii) to use the informed evidence for a formative study for intervention development on hypertension management and control among hypertensive patients. Hence, the objective of this study is to determine various healthcare personnel in delivering interventions as recommended by Disease Control Priorities (DCP3) strategies to manage and control high blood pressure among hypertensive patients.

\section{Description of Methodology}

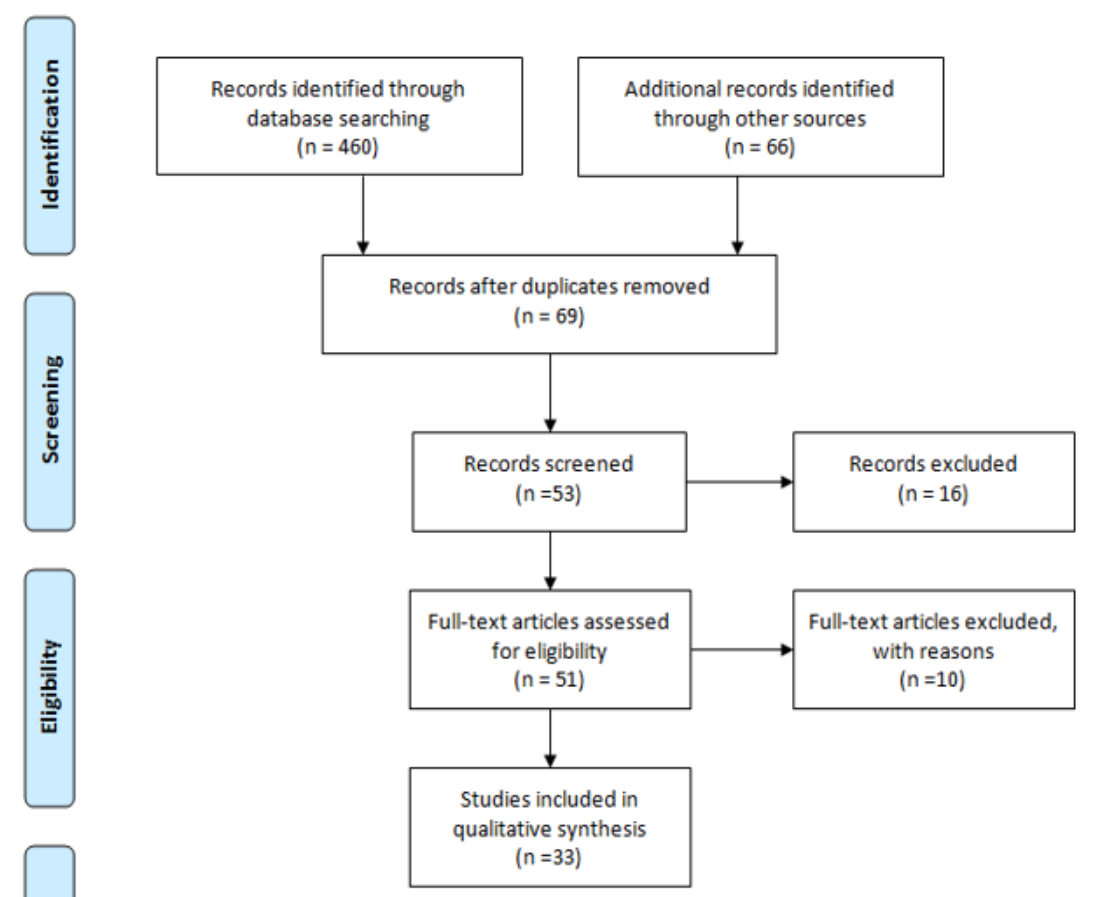

Figure 1: PRISMA Flow Chart $(\mathrm{N}=526)$. 
The guidance for conducting this desk review was adapted from a toolkit published by WHO and UNHCR (2012) and Save the children. For the desk review, secondary analysis was conducted of academically available and existing literature regarding hypertension management and control from 2015 to 2019 using PubMed and Google Scholar. The searched strategy included MeSH terms including "hypertension OR high blood pressure OR elevated or raised blood pressure". The inclusion criteria for DCP3 strategies on hypertension management and control was based on WHO's 25 by 25 identified key targets out of which 05 risk factors for cardiovascular diseases have been considered (sodium intake, use of tobacco, physical inactivity, high blood pressure and obesity) for this review [3]. The searching strategy included "Disease Control Priorities OR DCP3 AND strategies OR interventions AND sodium intake OR salt AND tobacco OR smoking AND physical inactivity OR physical activity OR sedentary AND body mass index OR BMI AND obesity AND healthcare provider OR physicians OR doctors OR cardiologist AND nurse OR nursing staff OR registered nurse or RN OR nursing staff AND paramedics OR technicians AND hypertensive patients". We selected 51 articles out of 526 article using MeSH terms (Figure 1). Total 526 numbers of articles were retrieved for the purpose of analysis; cleaning was done and duplication of researches was removed. The selected articles were then analyzed to synthesize available evidence and hence content of the desk review was prepared in accordance with the study objective.

\section{Results}

\section{Study Selection and Characteristics}

This review consists of available evidence for blood pressure reduction interventions delivered by physicians, pharmacists, nurses and other health professionals (Table1). Five key components of DCP3 strategies were focused which comprised of interventions for sodium intake $(n=140)$, smoking cessation $(n=160)$, physical activity, obesity and BMI $(n=226)$. Only hypertensive patients were included as the target population rather than individuals and general population for BP reduction interventions. Thus, a total of 51 articles were screened and included in thematic analysis.

Table 1: Studies on Intervention Delivered by Healthcare Personnel for Hypertension $(\mathrm{N}=33)$.

\begin{tabular}{|c|c|c|}
\hline S. No. & Interventions & Healthcare Personnel \\
\hline 1. & Use of sodium in diet & $\begin{array}{c}\text { Physician led strategies } \\
\text { Community healthcare workers }\end{array}$ \\
\hline 2. & Smoking cessation & $\begin{array}{c}\text { Physician led strategies } \\
\text { Pharmacist led strategies }\end{array}$ \\
\hline 3. & $\begin{array}{c}\text { Lifestyle changes \& } \\
\text { Physical exercise }\end{array}$ & $\begin{array}{c}\text { Physician led strategies } \\
\text { Community healthcare workers }\end{array}$ \\
\hline
\end{tabular}

\section{Physician-led interventions}

Use of Sodium: Blood pressure control can be achieved by reducing salt consumption in diet which can prevent and delay future complications of hypertension. Interventions can be effective when reduction in sodium is not only limited to meals, preparing and consuming food but when hypertensive patients are counseled effectively about improving blood pressure by low sodium in diet [8]. Literature also indicates salinity of drinking water or drinking ground water, which is high in salt, is also been implicated in causing high blood pressure [9]. Along with these public health measures, in food particularly 'bread' has been considered for reducing salt content so as to achieve blood pressure prevention and control in populations [10,11]. In Western countries, salt restriction strategies were posed on processed foods by physicians and public health experts. A study showed to prevent onset of cardiovascular diseases in the Dutch population. Evidence suggested that saltrestriction activities can help to avert hypertension, but more attention should be paid to the demand of consumers of processed food containing low salt in it [12]. Although, other countries despite having same cultural preference of added salt in foods did not yielded significant results from such initiatives. In China, reduction in cooking salt was achieved from salt-restriction spoons and salt substitutes strategies [13-15] whereas; evidence from Australia showed significant decrease in consumption from salt-reduction strategies. but these researches fostered health education as integral to success of lowering sodium in diet which ultimately help to avert hypertension, cardiovascular diseases and related complications [16,17]. In addition, salt sensitive individuals who façade blood pressure sensitivity have also been ascribed as highrisk population which can be target for blood pressure control interventions [18].

\section{Smoking Cessation}

The prevalence of smoking is maintained at past rates in LMICs, but tobacco toll is on rise which indicates the gap for tobacco control and promotion initiatives [19]. Findings from a study which investigated the effectiveness of intervention comprising of smoking cessation, explicated that quitting smoking has the most beneficial effects among current smokers [20]. In a similar physician-led study, smoking cessation related intervention was given to uninsured patients, but the findings showed statistically non-significant results for counseling and medication. The results elucidated ethnic disparities in receiving the intervention despite of health insurance coverage among patients. Further, the study also showed that safety net clinics, qualified health centers' and rural health centers can be health sites for delivering smoking cessation intervention thereby covering mass population [21]. Even continuous efforts for smoking cessation strategies are necessary for a long duration as evidence shows that among men prolongation of smoking cessation tends to progressively increase the blood pressure resulting in hypertension [22].

\section{Lifestyle Changes}

Studies that focused on lifestyle changes have been found useful in delivering interventions to hypertensive patients. Interventions 
for blood pressure reduction have been successful in attaining the recommended BP among hypertensive patients. However; evidence shows that single-session interventions versus multi-component interventions are both effective in reducing BP but the findings were statistically non-significant [23]. Comparative effectiveness of lifestyle modifications such as yoga on prevention of hypertension has been found as an effective strategy and reduces the 10-year risk of CVD [20]. Regarding obesity which is a risk factor for hypertension and is implicated in higher levels of BMI which therefore increases the blood pressure despite presence of other risk factors [24]. Research shows that exercise has impeccable impact on cardiac health and in lowering blood pressure. A moderate exercise of up to 30 minutes as recommended by American Heart Association prevents complications of high blood pressure. Hypertensive patient's ongoing treatment, mode, pattern and timing of exercise can be personalized as compared to less efficient exercises to reduce the risk of CVD events [25]. To consider long-term changes in preventing hypertension related complications, lifestyle modification must be recommended particularly among young adults [26].

\section{Pharmacist-led Interventions}

Findings from PURE study elucidated that individuals who mainly smoke or have hypertension or history of cardiovascular diseases can have increased risk of CVD events up to 84\% [27]. Pharmacist-led smoking cessation programs have been delivered in the world. A similar study tested the effect of a structured smoking cessation program with three follow-up sessions and showed a greater decrease in smoking cessation rates at 12 months of time period [28]. According to the findings of a similar systematic review in which approximately nine studies showed an intervention effect for group counseling as compared to individual counseling [29].

\section{Health Professionals}

Task shifting is essential with overloaded health systems in the world which mainly involves nurses, paramedics and community health workers (CHWs). Scarcity of resources and understaffed healthcare system necessitates cost-effective interventions in LMICs for blood pressure control [30]. Skilled human resource such as community health workers (CHWs) is among the hugely important cadre in mobilizing communities. CHWs have been indicated to work for hypertension, its detection and treatment after adequate training and supervision as they can effectively integrate into the existing health system and are known in the communities. They have been involved in NCD programs to support and provide advices for avoiding risk factors of noncommunicable diseases and for adopting healthy lifestyles such as physical exercise and diet [31]. Healthcare professionals such as registered dietitian and nutritionist were mainly involved in delivering interventions for counseling on low sodium intake $[10,11]$. Regarding tobacco cessation programs, evidence showed that CHWs are integral to NCD related programs for primary prevention of cardiovascular diseases particularly in LMICs. They have been designated as backbone of primary healthcare services and are found to be relatively inexpensive than other cadres for blood pressure, diabetes and most of NCDs in the communities [32]. Whereas community pharmacist has been studied for smoking cessation interventions. The study findings suggests that pharmacist's role in the community revolves around identity, capability, motivation, clinical confidence and in public trust which infers that they can be involved in behavioral interventions such as tobacco cessation among hypertensive patients [33]. Moreover, family support and involvement are also considered for managing hypertensive patients. Evidence suggests that involving family improves compliance of patients to medicines. For the management and treatment of hypertension, family member-based supervised therapy can be significantly effective in improving adherence to medications and BP monitoring of hypertensive patients [34].

\section{Conclusion}

Worldwide, physician-led interventions specifically for sodium intake have been studied for lowering blood pressure and hypertension control followed by smoking cessation and physical activity. Scarcity in available evidence exists for nurses; however, pharmacists and CHWs are being mainly included for delivering supervised interventions and in task shifting roles. A less available data exists in literature for multi-component $\mathrm{BP}$ reduction interventions delivered by physicians. It is therefore essential that population-level interventions must be focused for BP control and prevention of NCDs risk. Rural population must also be targeted in implementing locally adapted BP reduction strategies. Whereas considering understaffed health system in LMICs, other healthcare professionals can be utilized to implement awareness and behavioral interventions based on DCP3 strategies in communities.

\section{Acknowledgments}

All authors thank Dr. Malik Muhammad Safi from Ministry of National Health Services, Regulation \& Coordination for providing valuable guidance for interviewing study participants.

\section{Funding}

No funding was received to conduct this review.

\section{Author Contribution}

Subhana Akber Khan conceived the research idea, developed the initial manuscript and conducted the analysis. Dr. Saadullah Afridi, Dr. Zeeshan Kibria contributed to the writing of manuscript. Dr. Assad Hafeez, Dr. Siham Sikander, Dr. Syed Fawad Mashadi and Dr. Ejaz Ahmad Khan review and approved the final manuscript. 


\section{Conflict of Interest}

All authors declare no conflict of interest.

\section{References}

1. Mensah GA, Roth GA, Fuster V (2019) The Global Burden of Cardiovascular Diseases and Risk Factors: 2020 and Beyond. J Am Coll Cardiol 74(20): 2529-2532.

2. (2019) Control High Blood Pressure | 6|18 Initiative | CDC [Internet].

3. (2016) AHA/WHF Scientific Statement e674.

4. Lee ES, Vedanthan R, Jeemon P, Kamano JH, Kudesia P, et al. (2019) Quality Improvement in Cardiovascular Disease Care. PLoS One 11(6): e0157036.

5. Carnagarin R, Matthews V, Gregory C, Schlaich MP (2018) Pharmacotherapeutic strategies for treating hypertension in patients with obesity. Expert Opin Pharmacother 19(7): 643-651.

6. Herrett E, Gadd S, Jackson R, Bhaskaran K, Williamson E, et al. (2019) Eligibility and subsequent burden of cardiovascular disease of four strategies for blood pressure-lowering treatment: a retrospective cohort study. 394: 663 .

7. Webb M, Fahimi S, Singh GM, Khatibzadeh S, Micha R, et al. (2017) Cost effectiveness of a government supported policy strategy to decrease sodium intake: Global analysis across 183 nations. BMJ 356

8. Trieu K, Mcmahon E, Santos JA, Bauman A, Jolly KA, et al. (2017) Review of behaviour change interventions to reduce population salt intake. Int J Behav Nutr Phys Act 14(1): 17.

9. Naser AM, Unicomb L, Doza S, Ahmed KM, Rahman M, et al. (2017) Stepped-wedge cluster-randomised controlled trial to assess the cardiovascular health effects of a managed aquifer recharge initiative to reduce drinking water salinity in southwest coastal Bangladesh: Study design and rationale. BMJ Open 7(9): e015205.

10. Ruzicka M, Ramsay T, Bugeja A, Edwards C, Fodor G, et al. (2011) Does pragmatically structured outpatient dietary counselling reduce sodium intake in hypertensive patients? Study protocol for a randomized controlled trial. Trials 16: 273.

11. Cashman KD, Kenny S, Kerry JP, Leenhardt F, Arendt EK. (2019) "LowSalt" Bread as an Important Component of a Pragmatic Reduced-Salt Diet for Lowering Blood Pressure in Adults with Elevated Blood Pressure. Nutrients 11(8): 1725.

12. Ah Hendriksen M, Hoogenveen RT, Hoekstra J, Geleijnse JM, Boshuizen HC, et al. (2019) Potential effect of salt reduction in processed foods on health. Am J Clin Nutr 99(3): 446-453.

13. Chen J, Tian Y, Liao Y, Yang S, Li Z, et al. (2013) Salt Restriction-Spoon Improved the Salt Intake among Residents in China. PLoS One 8(11): e78963.

14. Hu J, Zhao L, Thompson B, Zhang Y, Wu Y (2018) Effects of salt substitute on home blood pressure differs according to age and degree of blood pressure in hypertensive patients and their families. Clin Exp Hypertens 40(7): 664-672.

15. Mcmahon E, Webster J, O’dea K, Brimblecombe J (2015) Dietary sodium and iodine in remote Indigenous Australian communities: will saltreduction strategies increase risk of iodine deficiency? A cross-sectional analysis and simulation study. BMC Public Health 15: 1318.

16. Wang M, Moran AE, Liu J, Coxson PG, Penko J, et al. (2016) Projected Impact of Salt Restriction on Prevention of Cardiovascular Disease in China: A Modeling Study. PLoS One 11(2): e0146820.

17. Quader ZS, Cogswell ME, Fang J, Coleman King SM, Merritt RK (2017) Changes in primary healthcare providers' attitudes and counseling behaviors related to dietary sodium reduction, DocStyles 2010 and 2015. PLoS One 12(5): e0177693.
18. Chen ML, Huang TP, Chen TW, Chan HH, Hwang BF (2018) Interactions of Genes and Sodium Intake on the Development of Hypertension: A Cohort-Based Case-Control Study. Int J Environ Res Public Health 15(6): 1110.

19. Reitsma MB, Fullman N, Ng M, Salama JS, Abajobir A, et al. (2017) Smoking prevalence and attributable disease burden in 195 countries and territories, 1990-2015: a systematic analysis from the Global Burden of Disease Study 2015. Lancet 389(10082): 1885-1906.

20. Chu P, Pandya A, Salomon JA, Goldie SJ, Hunink Myriam MG (2016) Comparative Effectiveness of Personalized Lifestyle Management Strategies for Cardiovascular Disease Risk Reduction. J Am Heart Assoc 5(3): e002737.

21. Bailey SR, Heintzman J, Jacob RL, Puro J, Marino M (2018) Disparities in Smoking Cessation Assistance in US Primary Care Clinics. Am J Public Health 108(8): 1082-1090.

22. Lee DH, Ha MH, Kim JR, Jacobs DR (2001) Effects of Smoking Cessation on Changes in Blood Pressure and Incidence of Hypertension A 4-Year Follow-Up Study. Hypertension 37(2): 194-198.

23. Schoenthaler A, Luerassi L, Silver S, Odedosu T, Kong J, et al. (2016) Comparative Effectiveness of a Practice-Based Comprehensive Lifestyle Intervention vs. Single Session Counseling in Hypertensive Blacks. Am J Hypertens 29(2): 280-287.

24. Landi F, Calvani R, Picca A, Tosato M, Martone AM, et al. (2018) Body Mass Index is Strongly Associated with Hypertension: Results from the Longevity Check-Up 7+ Study. Nutrients 10(12): 1976.

25. Tian D, Meng J (2019) Exercise for Prevention and Relief of Cardiovascular Disease: Prognoses, Mechanisms, and Approaches. Oxid Med Cell Longev 2019: 3756750.

26. Williamson W, Huckstep OJ, Frangou E, Mohamed A, Tan C, et al. (2018) Trial of exercise to prevent HypeRtension in young adults (TEPHRA) a randomized controlled trial: study protocol. BMC Cardiovasc Disord 18(1): 208.

27. Schwalm JD, Mckee M, Huffman MD, Yusuf S (2016) Resource Effective Strategies to Prevent and Treat Cardiovascular Disease. Circulation 133(8): 742-755

28. Saffouh M, Hajj E, Kheir N, Al Mulla AM, Shami R, et al. (2017) Effectiveness of a pharmacist-delivered smoking cessation program in the State of Qatar: a randomized controlled trial. BMC Public Health 17(1): 215.

29. Thomas R Fanshawe, William Halliwell, Nicola Lindson, Paul Aveyard, Jonathan Livingstone Banks, et al. (2017) Tobacco cessation interventions for young people (Review). Cochrane Database Syst Rev 11(11): CD003289.

30. Jeemon P, Anand TN, Maria Joseph L, Geetha A V, Prabhakaran D (2019) Task sharing with non-physician health-care workers for management of blood pressure in low-income and middle-income countries: a systematic review and meta-analysis. The Lancet Global Health.

31. Mishra SR, Neupane D, Preen D, Perry HB (2012) Development cooperation for health: reviewing a dynamic concept in a complex global aid environment.

32. Jeet G, Thakur JS, Prinja S, Singh M (2017) Community health workers for non-communicable diseases prevention and control in developing countries: Evidence and implications. PLoS One 12(7): e0180640.

33. Greenhalgh T, Macfarlane F, Steed L, Walton R (2016) What works for whom in pharmacist-led smoking cessation support: realist review. BMC Med 14(1): 209.

34. Shen Y, Peng X, Wang M, Zheng X, Xu G, et al. (2017) Family memberbased supervision of patients with hypertension: a cluster randomized trial in rural China. J Hum Hypertens 31(1): 29-36. 